\title{
Generalized Chen-Wu type cosmological model
}

\author{
Moncy V. John* and K. Babu Joseph \\ Department of Physics, Cochin University of Science and Technology, \\ Kochi 682022, India.
}

\begin{abstract}
Recent measurements require modifications in conventional cosmology by way of introducing components other than ordinary matter into the total energy density in the universe. On the basis of some dimensional considerations in line with quantum cosmology, Chen and Wu [W. Chen and Y. Wu, Phys. Rev. D 41, 695 (1990)] have argued that an additional component, which corresponds to an effective cosmological constant $\Lambda$ must vary as $a^{-2}$ in the classical era. Their decaying- $\Lambda$ model assumes inflation and yields a value for $q_{0}$, which is not compatible with observations. We generalize this model by arguing that the Chen- $\mathrm{Wu}$ ansatz is applicable to the total energy density of the universe and not to $\Lambda$ alone. The resulting model, which has a coasting evolution (i.e., $a \propto t$ ), is devoid of the problems of horizon, flatness, monopole, cosmological constant, size, age and generation of density perturbations. However, to avoid serious contradictions with big bang nucleosynthesis, the model has to make the predictions $\Omega_{m}=4 / 3$ and $\Omega_{\Lambda}=2 / 3$, which in turn are at variance with current observational values.
\end{abstract}

PACS No(s): 98.80.-k

*Permanent address: Department of Physics, St. Thomas College, Kozhencherri 689641, Kerala, India. e-mail: moncy@stthom.ernet.in 
Recent measurements [1] of the cosmic deceleration parameter, which point to the need of having some new energy density in the present universe, in addition to the usual relativistic/nonrelativistic matter density have caused some sensation [2]. Several other measurements like that of the combination of the Hubble parameter $H_{0}$ and the age $t_{0}$ of the present universe, gravitational lensing, etc., also indicate such a possibility. Candidates for such an additional component include vacuum energy with density $\rho_{\Lambda}$ (identical to that due to a cosmological constant $\Lambda$, with equation of state $p_{\Lambda}=-\rho_{\Lambda}$ ) and "quintessence" [3] with density $\rho_{q}$ (with a general equation of state $p_{q}=w \rho_{q} ;-1<w<0$ - examples are fundamental fields and macroscopic objects such as light, tangled cosmic strings), the former being considered often in the literature. The above observations specifically show that if the new component is $\rho_{\Lambda}$, then its magnitude should be comparable to that of matter density $\rho_{m}$. Decaying vacuum cosmologies [4-8] (and references therein) are phenomenological models, which conceive a time-varying $\Lambda$ as an attempt to describe how $\rho_{\Lambda}$ attains such small values in the present universe. In this report, we study one of the pioneering decaying vacuum models [7] and suggest an alternative scenario which is conceptually more sound. Though the resulting model faces some serious problems when concrete theoretical predictions, either on nucleosynthesis or on the density parameters $\Omega_{m}$ and $\Omega_{\Lambda}$ are compared with observations, it has several positive features and raises certain fundamental issues which invite serious consideration.

First we recall that Chen and $\mathrm{Wu}$ [7], while introducing their widely discussed model mentioned above, have made an interesting argument in favor of an $a^{-2}$ variation of the effective cosmological constant on the basis of some dimensional considerations in line with quantum cosmology. Their reasoning is as follows: Since there is no other fundamental energy scale available, one can always write $\rho_{\Lambda}$, the energy density corresponding to the effective cosmological constant as the Planck density $\left(\rho_{p l}=c^{5} / \hbar G^{2}=5.158 \times 10^{94} \mathrm{gm} \mathrm{cm}^{-3}\right.$ ) times a dimensionless product of quantities. Assuming that $\rho_{\Lambda}$ varies as a power of the scale factor $a$, the natural ansatz is

$$
\rho_{\Lambda} \propto \frac{c^{5}}{\hbar G^{2}}\left[\frac{l_{p l}}{a}\right]^{n},
$$

where $l_{p l}=\left(\hbar G / c^{3}\right)^{1 / 2}=1.616 \times 10^{-33} \mathrm{~cm}$ is the Planck length. The authors argue that $n=2$ is a preferred choice. It is easy to verify that $n<2$ (or $n>2$ ) will lead to a negative (positive) power of $\hbar$ appearing explicitly on the right hand side of the above equation. Such an $\hbar$-dependent $\rho_{\Lambda}$ would be quite unnatural in the classical Einstein equation for cosmology, much later than the Planck time. However, it shall be noted that $n=2$ is just right to survive the semiclassical limit $\hbar \rightarrow 0$. This choice is further substantiated by noting that $n \leq 1$ or $n \geq 3$ would lead to a value of $\rho_{\Lambda}$ which violates all observational bounds. Thus the Chen-Wu ansatz is

$$
\rho_{\Lambda}=\frac{\gamma}{8 \pi G a^{2}}
$$

where $\gamma$ is a phenomenological constant parameter. (Here onwards we set $\hbar=c=k_{B}=1$, 
except when stating explicit results). Assuming that only the total energy-momentum is conserved, they obtain, for the relativistic era,

$$
\rho_{r}=\frac{A_{1}}{a^{4}}+\frac{\gamma}{8 \pi G a^{2}} \equiv \rho_{r}^{\text {cons. }}+\rho_{r}^{\text {noncons }}
$$

and for the nonrelativistic era,

$$
\rho_{n r}=\frac{A_{2}}{a^{3}}+\frac{2 \gamma}{8 \pi G a^{2}} \equiv \rho_{n r}^{\text {cons. }}+\rho_{n r}^{\text {noncons. }},
$$

where $A_{1}$ and $A_{2}$ are to be positive. The Chen-Wu model thus differs from the standard model in that it has a decaying cosmological constant and that the matter density has conserving and nonconserving parts [given by the first and second terms respectively in the right hand sides of Eqs. (3) and (4)]. By choosing $\gamma$ appropriately, they hope to arrange $\rho_{\Lambda}$ and the nonconserving parts in $\rho_{r}$ and $\rho_{n r}$ to be insignificant in the early universe so that the standard model results like nucleosynthesis are undisturbed. But for the late universe, it can have many positive features like providing the missing energy density in the flat and inflationary models, etc.. The model predicts creation of matter, but the authors argue that the creation rate is small enough so that it is inaccessible to observations.

The important criticisms one can raise in this regard are the following: Conversely to the requirement that the conserving part of matter density dominate the early universe (for the standard model results to remain undisturbed), one can deduce that in their model, the standard model results are applicable only to the same part of matter density. The nonconserving parts are, in fact, created almost entirely in the late universe. But the abundance of light nuclei etc. are verified for the present universe and this implies that the conserving part is still substantial. This in turn will create some problem with observations. For example, let us assume that the present era is nonrelativistic and $\rho_{n r}^{\text {cons }}$ is at least equal to $\rho_{n r}^{\text {noncons. }}$. Since the vacuum density is only one-half the latter quantity [See Eqs. (2) and ([1)], for a $k=0$ universe in which $\Omega_{m}+\Omega_{\Lambda}=1$, the deceleration parameter at present will be $q_{0}=\left(\Omega_{m} / 2\right)-\Omega_{\Lambda}=0.2$. This is not compatible with the observations mentioned earlier [1].

Also, since it is conceived that their model is not different from the standard model in the early universe, to avoid the cosmological problems, they have to assume the occurrence of inflation, which in turn is driven by the vacuum energy. But they apply their ansatz only to the late-time vacuum energy density (which corresponds to the cosmological constant) and not to that during inflation. The stress energy associated with the vacuum energy is identical to that of a cosmological constant and it is not clear how they distinguish them while applying the ansatz.

Lastly, it can genuinely be asked whether $\rho_{\Lambda}$ is the only quantity to which the Chen-Wu ansatz be applied. An equation analogous to (11) can be written for any kind of energy density by using a similar reasoning and it can be argued that $n=2$ is a preferred choice for each one of them in the late universe. Certainly, this will bring in some fundamental 
issues which need serious consideration, but there is a priori no reason to forbid such an investigation.

In this report, we present a cosmological model by applying the Chen-Wu ansatz to the total energy density $\tilde{\rho}$ of the universe, in place of the vacuum density alone. If the Chen-Wu argument is valid for $\rho_{\Lambda}$, then it should be valid for $\tilde{\rho}$ too. In fact, this ansatz is better suited to $\tilde{\rho}$ rather than to $\rho_{\Lambda}$, since the Planck era is characterized by the Planck density for the universe, above which quantum gravity effects become important. Hence we modify the ansatz to write

$$
\tilde{\rho}=A \frac{c^{5}}{\hbar G^{2}}\left[\frac{l_{p l}}{a}\right]^{n},
$$

where $A$ is a positive dimensionless constant. As indicated above, when $\tilde{\rho}$ is the sum of various components and each component is assumed to vary as a power of the scale factor $a$, then the Chen-Wu argument can be applied to conclude that $n=2$ is a preferred choice for each component. Violating this will force the inclusion of $\hbar$-dependent terms in $\tilde{\rho}$, which would look unnatural in a classical theory. Not only for the Chen and $\mathrm{Wu}$ model, in all of FRW cosmology, this argument may be used to forbid the inclusion of substantial energy densities which do not vary as $a^{-2}$ in the classical epoch.

At first sight, this may appear as a grave negative result. But let us face it squarely and proceed to the next logical step of investigating the implications of an $a^{-2}$ variation of $\tilde{\rho}$. If the total pressure in the universe is denoted as $\tilde{p}$, then the above result that the conserved quantity $\tilde{\rho}$ in the FRW model varies as $a^{-2}$ implies $\tilde{\rho}+3 \tilde{p}=0$. This will lead to a coasting cosmology (i.e., $a \propto t$ ). Components with such an equation of state are known to be strings or textures [10]. Though such models are considered in the literature, it would be unrealistic to consider the present universe as string-dominated. A crucial observation which makes our model with $\tilde{\rho}$ varying as $a^{-2}$ realistic is that this variation leads to string-domination only if we assume $\tilde{\rho}$ to be unicomponent. Instead, if we assume, as done in inflationary, Chen and Wu and many other models (Friedmann-Lamaitre-Robertson-Walker cosmologies) that $\tilde{\rho}$ consists of parts corresponding to relativistic/ nonrelativistic matter (with equation of state $p_{m}=w \rho_{m}$ where $w=1 / 3$ for relativistic and $w=0$ for nonrelativistic cases) and also to a time-varying cosmological constant (with equation of state $p_{\Lambda}=-\rho_{\Lambda}$ ), i.e., if we assume,

$$
\tilde{\rho}=\rho_{m}+\rho_{\Lambda}, \quad \tilde{p}=p_{m}+p_{\Lambda},
$$

then the condition $\tilde{\rho}+3 \tilde{p}=0$ will give

$$
\frac{\rho_{m}}{\rho_{\Lambda}}=\frac{2}{1+3 w} .
$$

In other words, the modified Chen-Wu ansatz leads to the conclusion that if the universe contains matter and vacuum energies, then vacuum energy density should be comparable to matter density. This, of course, will again lead to a coasting cosmology, but this time a 
realistic one. (The Ozer-Taha model [4] in its relativistic era and the models in [5, 8] are approximately some such models, but they start from different sets of assumptions.)

$\rho_{m}$ or $\rho_{\Lambda}$, which varies as $a^{-2}$, may sometimes be mistaken for strings but it should be noted that the equations of state we assumed for these quantities are different from that for strings and are what they ought to be to correspond to matter density and vacuum energy density respectively. It is true that components with equations of state $p=w \rho$ should obey $\rho \propto a^{-3(1+w)}$, but this is valid when those components are separately conserved. In our case, we have only assumed that the total energy density is conserved and not the parts corresponding to $\rho_{m}$ and $\rho_{\Lambda}$ separately. Hence, as in the Chen-Wu model, there can be creation of matter from vacuum, but we shall show later in this report that again the present creation rate is too small to make any observational consequences.

The solution to the Einstein equations in an FRW model with $\tilde{\rho}+3 \tilde{p}=0$, for all the three cases $k=0, \pm 1$, is the coasting evolution

$$
a(t)=m t
$$

where $m$ is some proportionality constant. The total energy density is then

$$
\tilde{\rho}=\frac{3}{8 \pi G} \frac{\left(m^{2}+k\right)}{a^{2}} .
$$

Comparing this with (司) (with $n=2$ ), we get $m^{2}+k=8 \pi A / 3$. We shall now show that this simple picture of the universe is devoid of many of the cosmological problems encountered in the standard model.

First let us consider the horizon problem. A necessary condition for the solution of this problem is [12] $a\left(t_{s}\right) \int_{t_{p l}}^{t_{s}} d t / a(t)>\left[a\left(t_{s}\right) / a\left(t_{0}\right)\right] H_{0}^{-1}$, where $t_{s}$ is the time by which the horizon problem is solved. Using our expression (8) for $a(t)$, this condition gives $t_{s} \geq e t_{p l}$. Thus shortly after the Planck era, the horizon problem is solved in this model. Since causality is established at such early times, the monopole problem will also disappear.

The predictions regarding the age of the universe in the model is obvious from Eq. (8). Irrespective of the value of $m$, we get the combination $H_{0} t_{0}$ as equal to unity, which is well within the bounds. Thus there is no age problem in this model. We can legitimately define the critical density as $\rho_{c} \equiv(3 / 8 \pi G)\left(\dot{a}^{2} / a^{2}\right)$, so that Eq. (9) gives

$$
\tilde{\Omega} \equiv \frac{\tilde{\rho}}{\rho_{c}}=\left[1-\frac{3 k}{8 \pi A}\right]^{-1} .
$$

As in the standard model, we have $\tilde{\Omega}=1$ for $k=0$ and $\tilde{\Omega}>1(\tilde{\Omega}<1)$ for $k=+1$ $(k=-1)$. But unlike the standard model, $\tilde{\Omega}$ is a constant in time. This is not surprising; in an FRW model with total energy density $\tilde{\rho}$, one can always write the time-time component of Einstein equation in the form

$$
\tilde{\Omega}-1=\left[\frac{8 \pi G}{3} \frac{\tilde{\rho} a^{2}}{k}-1\right]^{-1} .
$$


When $\tilde{\rho}$ varies $a^{-3}$ or $a^{-4}$, the flatness problem appears and the reason can be understood from this equation. But in the present case, since $\tilde{\rho}$ varies as $a^{-2}, \tilde{\Omega}$ will remain a constant. Using Eqs. (6) and (7), we get

$$
\Omega_{m} \equiv \frac{\rho_{m}}{\rho_{c}}=\frac{2 \tilde{\Omega}}{3(1+w)}, \quad \Omega_{\Lambda} \equiv \frac{\rho_{\Lambda}}{\rho_{c}}=\frac{(1+3 w) \tilde{\Omega}}{3(1+w)} .
$$

For the matter dominated era, the predictions are $\Omega_{m}=2 \tilde{\Omega} / 3$ and $\Omega_{\Lambda}=\tilde{\Omega} / 3$. Note that also the density parameter $\Omega_{m}$ is time-independent and hence there is no flatness problem in this model. As mentioned above, the model predicts that the energy density corresponding to the cosmological constant is comparable with matter density and this solves the cosmological constant problem too. It can also be seen that according to the model, the observed universe, characterised by the present Hubble radius has a size equal to the Planck length at the end of Planck epoch and this indicates that the problem with the size of the universe does not appear here. For the investigation of other problems, we have to study the thermal evolution of the universe as envisaged in the model.

In the early relativistic era, temperature $T$ is associated with the relativistic matter density $\rho_{r}$ as $\rho_{r}=\left(\pi^{2} / 30\right) N(T) T^{4}$, where $N(T)$ is the effective number of spin degrees of freedom at temperature $\mathrm{T}$. In the present model,

$$
\rho_{r}=\frac{3 \tilde{\Omega}}{8 \pi G} \frac{1}{(\sqrt{2} t)^{2}} .
$$

This gives

$$
T=\left[\frac{3}{8 \pi G} \frac{30 \tilde{\Omega}}{\pi^{2} N}\right]^{1 / 4} \frac{1}{(\sqrt{2} t)^{1 / 2}} .
$$

These expressions may be compared with the corresponding expressions in the standard model:

$$
\begin{gathered}
\rho_{s . m .}=\frac{3}{8 \pi G} \frac{1}{(2 t)^{2}}, \\
T_{s . m .}=\left[\frac{3}{8 \pi G} \frac{30}{\pi^{2} N}\right]^{1 / 4} \frac{1}{(2 t)^{1 / 2}} .
\end{gathered}
$$

Considering the fact that according to observation $\tilde{\Omega}^{1 / 4}$ is close to unity, it can be seen that the values of $\rho_{r}$ and $T$ attained at time $t$ in the standard model are attained at time $\sqrt{2} t$ in the present model. Thus the thermal history in the present model can be expected to be nearly the same as that in the standard model. But the time-dependence of the scale factor is different in our model and this helps to solve the cosmological problems.

So far we have considered $\tilde{\Omega}$ to be a free parameter, related by Eq. (10) to the constant $A$, which in turn is to be understood to come from some deep quantum cosmological theory. 
An interesting way to estimate the constant $\tilde{\Omega}$ is to consider the implications of the model for nucleosynthesis [9]. From (13) and (15), one can deduce that the Hubble parameter in the present model is related to that in the standard model according to $H=\sqrt{2 / \tilde{\Omega}} H_{s . m}$. This modifies the ratio of interaction rate to Hubble parameter as $\Gamma / H=\sqrt{\tilde{\Omega} / 2} \Gamma / H_{s . m}$. To avoid any variation of the freezing temperature with that in the successful standard model, one has to accept a value $\tilde{\Omega} \approx 2$. This leads us to the predictions $\Omega_{m} \approx 4 / 3$ and $\Omega_{\Lambda} \approx 2 / 3$, which are in contradiction with the recent measurements [1] since the corresponding point is outside the error ellipses in the $\Omega_{m}-\Omega_{\Lambda}$ plot. This discrepancy with observation is a serious problem which requires detailed analysis and refinement in the model.

The possibility of the generation of density perturbations on scales well above the present Hubble radius, in the interval between the Planck time $t_{p l}$ and the time of decoupling $t_{d e c}$ can be studied by evaluating the communication distance light can travel between these two times [1]. In the present model, $d_{c o m m}\left(t_{p l}, t_{d e c}\right)=a_{0} \int_{t_{p l}}^{t_{d e c}} d t / a(t)=0.627 \times 10^{6} \mathrm{Mpc}$, where we have used $t_{d e c} \approx 10^{13} \mathrm{~s}$, the same as that in the standard model. Thus the coasting evolution in this case has the communication distance between $t_{p l}$ and $t_{d e c}$ much larger than the present Hubble radius $(\approx 4000 \mathrm{Mpc})$ and hence it can generate density perturbations on scales of that order. It is interesting to note that Liddle [11] has precluded coasting evolution as a viable means to produce such perturbations and argued that only inflation $(\ddot{a}>0)$ can perform this task, thus "closing the loopholes" in the arguments of $\mathrm{Hu}$ et. al. [12]. But it is worthwhile to point out that his observations are true only for a model which coasts from $t_{p l}$ to $t_{n u c}$ (where $t_{n u c} \approx 1 \mathrm{~s}$ is the time of nucleosynthesis) and thereafter evolves according to the standard model. In our case, the evolution is coasting throughout the history of the universe and hence his objection is not valid.

A bonus point of the present approach, when compared to all the other aforementioned models may now be noted. In those models, the communication distance between $t_{n u c}$ and $t_{d e c}$, or for that matter the communication distance from any time after the production of particles (assuming this to occur at the end of inflation) to the time $t_{d e c}$ will be only around $200 h^{-1} \mathrm{Mpc}, 0.6<h<0.8$ [11]. Thus density perturbations on scales above the present Hubble radius cannot be generated in them in the period when matter is present. This is because inflation cannot enhance the communication distance after it. The only means to generate the observed density perturbations is then to resort to quantum fluctuations of the inflaton field. The present model is at a more advantageous position than the inflationary models in this regard since the communication distance between $t_{n u c}$ and $t_{d e c}$ in this case is $d_{\text {comm }}\left(t_{n u c}, t_{d e c}\right)=a_{p} \int_{t_{\text {nuc }}}^{t_{\text {dec }}} d t / a(t)=1.45 \times 10^{5} \mathrm{Mpc}$, which is much greater than the present Hubble radius. So we can consider the generation of the observed density perturbations as a late-time classical behavior too.

Lastly we check the rate of matter creation in the model. Assuming the present universe to be dominated by nonrelativistic matter, we can calculate the rate of creation per unit volume as $a^{-2} d\left(\rho_{m} a^{3}\right) /\left.d t\right|_{p}=\rho_{m 0} H_{0}$. This creation rate is only one-third of that in the steady state model. Creation of matter or radiation with an average rate given above will be inaccessible to test and does not pose a serious objection to the model. 
It was recently argued [3] that a smooth time-varying $\Lambda$ is ill defined and unstable and that the only valid way of introducing an additional energy component is to replace $\Lambda$ with a fluctuating, inhomogeneous component. (Such an energy component is the quintessence, mentioned in the introduction.) Notwithstanding this and other serious problems with observations (either the big bang nucleosynthesis or the prediction of density parameters), it is worth noting that if we take quantum cosmology seriously, generalizing the Chen-Wu ansatz is a logical conclusion and that it leads to a realistic cosmological scenario, which does not have many of the problems in the standard model, including that of the generation of density perturbations in the late classical epoch itself.

We acknowledge the valuable comments by the unknown referee, with thanks. MVJ is grateful to IUCAA, Pune for its hospitality, where part of this work was done.

\section{References}

[1] S. Perlmutter et al., astro-ph/9812133 (1998) (to be published in Ap. J.).

[2] L. M. Krauss, Sci. Am. 280, 52 (1999).

[3] R. R. Caldwell, R. Dave and P. J. Steinhardt, Phys. Rev. Lett. 80, 1582 (1998).

[4] M. Ozer and M. O. Taha, Phys. Lett. 171B, 363 (1986); Nucl. Phys. B287, 776 (1987); B. Ratra and P. J. E. Peebles, Phys. Rev. D 37, 3407 (1988); J. C. Carvalho, J. A. S. Lima and I. Waga, Phys. Rev. D 46, 2404 (1992); J. A. S. Lima and M. Trodden, Phys. Rev. D 53, 4280 (1996).

[5] J. Lopez and D. V. Nanopoulos, Mod. Phys. Lett. A11, 1 (1996).

[6] K. Coble, S. Dodelson and J. A. Frieman, Phys. Rev. D 55, 1851 (1997).

[7] W. Chen and Y. Wu, Phys. Rev. D 41, 695 (1990).

[8] M. V. John and K. Babu Joseph, Phys. Lett. 387B, 466; Class. Quantum Grav. 14, 1115 (1997).

[9] E. W. Kolb and M. S. Turner, The Early Universe (Addison-Wesley, Redwood City, 1990); J. V. Narlikar, Introduction to Cosmology (Cambridge University Press, Cambridge, 1993).

[10] M. Kamionkowski and N. Toumbas, Phys. Rev. Lett. 77, 587 (1996).

[11] A. R. Liddle, Phys. Rev. D 51, 5347 (1995).

[12] Y. Hu, M. S. Turner and E. J. Weinberg, Phys. Rev. D 49, 3830 (1994). 